\title{
Upper limits on the strength of periodic gravitational waves from PSR J1939+2134
}

B Allen ${ }^{39}$ and G Woan ${ }^{35}$ (for the LIGO Scientific Collaboration):

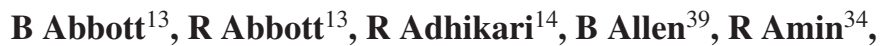
S B Anderson ${ }^{13}$, W G Anderson ${ }^{29}$, M Araya ${ }^{13}$, H Armandula ${ }^{13}$, F Asiri ${ }^{13}$, P Aufmuth ${ }^{31}$, C Aulbert ${ }^{1}$, S Babak ${ }^{7}$, R Balasubramanian ${ }^{7}, \mathbf{S}$ Ballmer $^{14}$, B C Barish ${ }^{13}$, D Barker ${ }^{15}$, C Barker-Patton ${ }^{15}$, M Barnes ${ }^{13}$, B Barr ${ }^{35}$, M A Barton ${ }^{13}$, K Bayer ${ }^{14}$, R Beausoleil ${ }^{26}$, K Belczynski $^{23}$, R Bennett $^{35}$, S J Berukoff ${ }^{1}$, J Betzwieser ${ }^{14}$, B Bhawal ${ }^{13}$, G Billingsley ${ }^{13}$, E Black $^{13}$, K Blackburn ${ }^{13}$, B Bland-Weaver ${ }^{15}$, B Bochner ${ }^{14}$, L Bogue $^{13}$, R Bork $^{13}$,

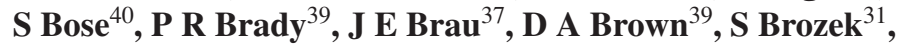
A Bullington ${ }^{26}$, A Buonanno ${ }^{6}$, R Burgess ${ }^{14}$, D Busby ${ }^{13}$, W E Butler ${ }^{38}$, R L Byer ${ }^{26}$, L Cadonati ${ }^{14}$, G Cagnoli ${ }^{35}$, J B Camp ${ }^{21}$, C A Cantley ${ }^{35}$, L Cardenas $^{13}$, K Carter ${ }^{16}$, M M Casey ${ }^{35}$, J Castiglione ${ }^{34}$, A Chandler ${ }^{13}$, J Chapsky $^{13}$, P Charlton ${ }^{13}$, S Chatterji ${ }^{14}$, Y Chen ${ }^{6}$, V Chickarmane ${ }^{17}$, D Chin $^{36}$, N Christensen ${ }^{8}$, D Churches ${ }^{7}, \mathbf{C ~ C o l a c i n o}{ }^{31,2}$, R Coldwell ${ }^{34}$, M Coles ${ }^{16}$, D Cook ${ }^{15}$, T Corbitt ${ }^{14}$, D Coyne ${ }^{13}$, J D E Creighton ${ }^{39}$, T D Creighton ${ }^{13}$, D R M Crooks ${ }^{35}$, P Csatorday ${ }^{14}$, B J Cusack ${ }^{3}$, C Cutler ${ }^{1}$,

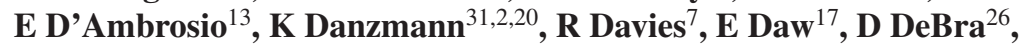
T Delker $^{34}$, R DeSalvo ${ }^{13}, \mathbf{S}$ Dhurandar ${ }^{12}$, M Díaz $^{29}, \mathbf{H}_{\text {Ding }}{ }^{13}$, R W P Drever ${ }^{4}$, R J Dupuis ${ }^{35}$, C Ebeling ${ }^{8}$, J Edlund ${ }^{13}$, P Ehrens ${ }^{13}$, E J Elliffe ${ }^{35}$, T Etzel ${ }^{13}$, M Evans ${ }^{13}$, T Evans ${ }^{16}$, C Fallnich ${ }^{31}$, D Farnham ${ }^{13}$, M M Fejer ${ }^{26}$, M Fine $^{13}$, L S Finn ${ }^{28}$, É Flanagan ${ }^{9}$, A Freise ${ }^{2}$, R Frey ${ }^{37}$, P Fritschel $^{14}$, V Frolov ${ }^{16}$, M Fyffe ${ }^{16}$, K S Ganezer ${ }^{5}$, J A Giaime ${ }^{17}$, A Gillespie ${ }^{13}$, K Goda ${ }^{14}$, G González ${ }^{17}$, S Goßler ${ }^{31}$, P Grandclément ${ }^{23}$, A Grant ${ }^{35}$, C Gray ${ }^{15}$, A M Gretarsson ${ }^{16}$, D Grimmett ${ }^{13}$, H Grote $^{2}$, S Grunewald ${ }^{1}$, M Guenther ${ }^{15}$, E Gustafson ${ }^{26}$, R Gustafson ${ }^{36}$, W O Hamilton ${ }^{17}$, M Hammond ${ }^{16}$, J Hanson ${ }^{16}$, C Hardham ${ }^{26}$, G Harry ${ }^{14}$, A Hartunian ${ }^{13}$, J Heefner ${ }^{13}$, Y Hefetz ${ }^{14}$, G Heinzel ${ }^{2}$, I S Heng ${ }^{31}$, M Hennessy ${ }^{26}$, N Hepler ${ }^{28}$, A Heptonstall ${ }^{35}$, M Heurs ${ }^{31}$, M Hewitson ${ }^{35}$, N Hindman ${ }^{15}$, P Hoang ${ }^{13}$, J Hough ${ }^{35}$, M Hrynevych ${ }^{13}$, W Hua ${ }^{26}$, R Ingley ${ }^{33}$, M Ito ${ }^{37}$, Y Itoh ${ }^{1}$, A Ivanov ${ }^{13}$, O Jennrich ${ }^{35}$, W W Johnson ${ }^{17}$, W Johnston ${ }^{29}$, L Jones ${ }^{13}$, D Jungwirth ${ }^{13}$, V Kalogera ${ }^{23}$,

E Katsavounidis ${ }^{14}$, K Kawabe ${ }^{20,2}$, S Kawamura ${ }^{22}$, W Kells ${ }^{13}$, J Kern ${ }^{16}$, A Khan ${ }^{16}$, S Killbourn ${ }^{35}$, C J Killow ${ }^{35}$, C Kim $^{23}$, C King ${ }^{13}$, P King ${ }^{13}$, S Klimenko ${ }^{34}$, P Kloevekorn ${ }^{2}$, S Koranda ${ }^{39}$, K Kötter ${ }^{31}$, J Kovalik $^{16}$, D Kozak ${ }^{13}$, B Krishnan ${ }^{1}$, M Landry ${ }^{15}$, J Langdale ${ }^{16}$, B Lantz ${ }^{26}$, R Lawrence ${ }^{14}$, A Lazzarini ${ }^{13}$, M Lei ${ }^{13}$, V Leonhardt ${ }^{31}$, I Leonor $^{37}$, K Libbrecht ${ }^{13}$, P Lindquist ${ }^{13}$, S Liu ${ }^{13}, \mathbf{J ~ L o g a n ~}^{13}$, M Lormand ${ }^{16}$, M Lubinski ${ }^{15}$, H Lück ${ }^{31,2}$, T T Lyons ${ }^{13}$, B Machenschalk ${ }^{1}$, M MacInnis ${ }^{14}$, M Mageswaran ${ }^{13}$, K Mailand ${ }^{13}$, W Majid ${ }^{13}$, M Malec ${ }^{31}$, F Mann ${ }^{13}$, A Marin ${ }^{14}$, S Márka ${ }^{13}$, E Maros ${ }^{13}$, J Mason ${ }^{13}$, K Mason ${ }^{14}$, 
O Matherny ${ }^{15}$, L Matone ${ }^{15}$, N Mavalvala ${ }^{14}, \mathbf{R}$ McCarthy $^{15}$,

D E McClelland ${ }^{3}$, M McHugh ${ }^{19}$, P McNamara $^{35}$, G Mendell ${ }^{15}$, S Meshkov ${ }^{13}, \mathbf{C}$ Messenger ${ }^{33}$, G Mitselmakher $^{34}$, R Mittleman $^{14}$, O Miyakawa ${ }^{13}$, S Miyoki $^{13}$, S Mohanty $^{1}$, G Moreno $^{15}$, K Mossavi $^{2}$,

B Mours ${ }^{13}$, G Mueller $^{34}, \mathbf{S}$ Mukherjee ${ }^{1}, \mathbf{J}$ Myers ${ }^{15}, \mathbf{S}$ Nagano $^{2}, \mathbf{T}$ Nash $^{10}$, H Naundorf ${ }^{1}, \mathbf{R}$ Nayak $^{12}$, G Newton ${ }^{35}, \mathbf{F}$ Nocera ${ }^{13}$, P Nutzman $^{23}$, T Olson ${ }^{24}$, B O'Reilly ${ }^{16}$, D J Ottaway ${ }^{14}$, A Ottewill ${ }^{19}$, D Ouimette ${ }^{13}$, H Overmier ${ }^{16}$, B J Owen ${ }^{28}$, M A Papa ${ }^{1}$, C Parameswariah ${ }^{16}$,

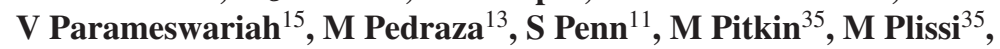
M Pratt ${ }^{14}$, V Quetschke ${ }^{31}$, F Raab ${ }^{15}$, H Radkins ${ }^{15}$, R Rahkola ${ }^{37}$, M Rakhmanov ${ }^{34}$, S R Rao ${ }^{13}$, D Redding ${ }^{13}$, M W Regehr ${ }^{13}$, T Regimbau ${ }^{14}$, K T Reilly ${ }^{13}$, K Reithmaier ${ }^{13}$, D H Reitze ${ }^{34}$, S Richman ${ }^{14}$, R Riesen ${ }^{16}$, K Riles $^{36}$, A Rizzi ${ }^{16}$, D I Robertson ${ }^{35}$, N A Robertson ${ }^{35,26}$, L Robison ${ }^{13}$, S Roddy ${ }^{16}$, J Rollins ${ }^{14}$, J D Romano ${ }^{29}$, J Romie ${ }^{13}$, H Rong ${ }^{34}$, D Rose ${ }^{13}$, E Rotthoff ${ }^{28}, \mathbf{S}$ Rowan ${ }^{35}$, A Rüdiger $^{20,2}, \mathbf{P}$ Russell $^{13}$, K Ryan ${ }^{15}$, I Salzman ${ }^{13}$, G H Sanders $^{13}$, V Sannibale ${ }^{13}$, B Sathyaprakash ${ }^{7}$, P R Saulson ${ }^{27}$, R Savage ${ }^{15}$, A Sazonov $^{34}, \mathbf{R}_{\text {Schilling }}{ }^{20,2}, \mathbf{K}_{\text {Schlaufman }}{ }^{28}$, V Schmidt $^{13}$, R Schofield ${ }^{37}$, M Schrempel ${ }^{31}$, B F Schutz ${ }^{1,7}$, P Schwinberg ${ }^{15}$, S M Scott ${ }^{3}$, A C Searle ${ }^{3}$, B Sears ${ }^{13}$, S Seel $^{13}$, A S Sengupta ${ }^{12}$, C A Shapiro ${ }^{28}$, P Shawhan ${ }^{13}$, D H Shoemaker ${ }^{14}$, Q Z Shu ${ }^{34}$, A Sibley ${ }^{16}$, X Siemens ${ }^{39}$, L Sievers $^{13}$, D Sigg ${ }^{15}$, A M Sintes ${ }^{1,32}$, K Skeldon ${ }^{35}$, J R Smith ${ }^{2}$, M Smith ${ }^{14}$, M R Smith ${ }^{13}$, P Sneddon ${ }^{35}$,

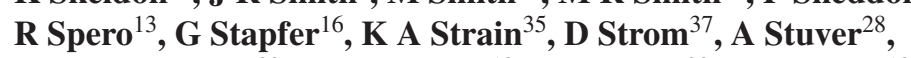
T Summerscales ${ }^{28}$, M C Sumner ${ }^{13}$, P J Sutton ${ }^{28}$, J Sylvestre ${ }^{13}$, A Takamori ${ }^{13}$, D B Tanner ${ }^{34}$, H Tariq $^{13}$, I Taylor ${ }^{7}$, R Taylor $^{13}$, K S Thorne ${ }^{6}$, M Tibbits ${ }^{28}, \mathbf{S}$ Tilav $^{13}$, M Tinto ${ }^{4}$, C Torres $^{29}$, C Torrie ${ }^{13,35}$, S Traeger ${ }^{31}, \mathbf{G}$ Traylor $^{16}, \mathbf{W}$ Tyler $^{13}$, D Ugolini ${ }^{30}, \mathbf{M}$ Vallisneri $^{6}$, M van Putten ${ }^{14}$, S Vass ${ }^{13}$, A Vecchio ${ }^{33}$, C Vorvick ${ }^{15}$, L Wallace ${ }^{13}$, H Walther ${ }^{20}$, H Ward ${ }^{35}$, B Ware ${ }^{13}$, K Watts $^{16}$, D Webber ${ }^{13}$, A Weidner ${ }^{20,2}, \mathbf{U}$ Weiland $^{31}, \mathbf{A}$ Weinstein ${ }^{13}, \mathbf{R}$ Weiss ${ }^{14}, \mathbf{H}$ Welling ${ }^{31}$, L Wen ${ }^{13}$, S Wen ${ }^{17}$, J T Whelan ${ }^{19}$, S E Whitcomb ${ }^{13}$, B F Whiting ${ }^{34}$, P A Willems ${ }^{13}$, P R Williams ${ }^{1}$, R Williams ${ }^{4}$, B Willke ${ }^{31,2}$, A Wilson ${ }^{13}$, B J Winjum ${ }^{28}$, W Winkler ${ }^{20,2}$, S Wise ${ }^{34}$, A G Wiseman ${ }^{39}$, G Woan $^{35}$,

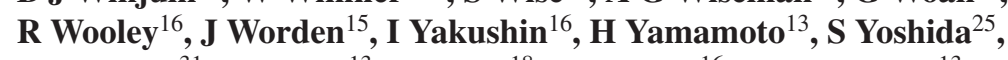
I Zawischa $^{31}$, L Zhang ${ }^{13}$, N Zotov ${ }^{18}$, M Zucker ${ }^{16}$ and J Zweizig ${ }^{13}$

\footnotetext{
${ }^{1}$ Albert Einstein Institut für Gravitationsphysik, D-14476 Golm, Germany

${ }^{2}$ Albert Einstein Institut für Gravitationsphysik, D-30157 Hannover, Germany

${ }^{3}$ Australian National University, Canberra, 0200, Australia

${ }^{4}$ California Institute of Technology, Pasadena, CA 91125, USA

${ }^{5}$ California State University Dominguez Hills, Carson, CA 90747, USA

${ }^{6}$ Caltech-CaRT, Pasadena, CA 91125, USA

${ }^{7}$ Cardiff University, Cardiff, CF2 3YB, UK

${ }^{8}$ Carleton College, Northfield, MN 55057, USA

${ }^{9}$ Cornell University, Ithaca, NY 14853, USA

${ }^{10}$ Fermi National Accelerator Laboratory, Batavia, IL 60510, USA

${ }^{11}$ Hobart and William Smith Colleges, Geneva, NY 14456, USA

12 Inter-University Centre for Astronomy and Astrophysics, Pune - 411007, India

${ }^{13}$ LIGO_California Institute of Technology, Pasadena, CA 91125, USA

${ }_{14}$ LIGO_Massachusetts Institute of Technology, Cambridge, MA 02139, USA

${ }^{15}$ LIGO Hanford Observatory, Richland, WA 99352, USA

${ }^{16}$ LIGO Livingston Observatory, Livingston, LA 70754, USA
} 
${ }^{17}$ Louisiana State University, Baton Rouge, LA 70803, USA

${ }^{18}$ Louisiana Tech University, Ruston, LA 71272, USA

${ }^{19}$ Loyola University, New Orleans, LA 70118, USA

${ }^{20}$ Max Planck Institut für Quantenoptik, D-85748, Garching, Germany

${ }^{21}$ NASA/Goddard Space Flight Center, Greenbelt, MD 20771, USA

${ }^{22}$ National Astronomical Observatory of Japan, Tokyo 181-8588, Japan

${ }^{23}$ Northwestern University, Evanston, IL 60208, USA

${ }^{24}$ Salish Kootenai College, Pablo, MT 59855, USA

25 Southeastern Louisiana University, Hammond, LA 70402, USA

${ }^{26}$ Stanford University, Stanford, CA 94305, USA

27 Syracuse University, Syracuse, NY 13244, USA

28 The Pennsylvania State University, University Park, PA 16802, USA

${ }^{29}$ The University of Texas at Brownsville and Texas Southmost College, Brownsville, TX 78520, USA

${ }^{30}$ Trinity University, San Antonio, TX 78212, USA

${ }^{31}$ Universität Hannover, D-30167 Hannover, Germany

32 Universitat de les Illes Balears, E-07071 Palma de Mallorca, Spain

${ }^{33}$ University of Birmingham, Birmingham, B15 2TT, UK

${ }^{34}$ University of Florida, Gainsville, FL 32611, USA

35 University of Glasgow, Glasgow, G12 8QQ, UK

${ }^{36}$ University of Michigan, Ann Arbor, MI 48109, USA

${ }^{37}$ University of Oregon, Eugene, OR 97403, USA

${ }^{38}$ University of Rochester, Rochester, NY 14627, USA

${ }^{39}$ University of Wisconsin-Milwaukee, Milwaukee, WI 53201, USA

${ }^{40}$ Washington State University, Pullman, WA 99164, USA

Received 2 October 2003

Published 9 February 2004

Online at stacks.iop.org/CQG/21/S671 (DOI: 10.1088/0264-9381/21/5/042)

\begin{abstract}
The first science run of the LIGO and GEO gravitational wave detectors presented the opportunity to test methods of searching for gravitational waves from known pulsars. Here we present new direct upper limits on the strength of waves from the pulsar PSR J1939+2134 using two independent analysis methods, one in the frequency domain using frequentist statistics and one in the time domain using Bayesian inference. Both methods show that the strain amplitude at Earth from this pulsar is less than a few times $10^{-22}$.
\end{abstract}

PACS numbers: $04.80 . \mathrm{Nn}, 95.55 . \mathrm{Ym}, 97.60 . \mathrm{Gb}, 07.05 . \mathrm{Kf}$

\title{
1. Introduction
}

The LIGO and GEO gravitational wave detectors performed their first science run, denoted S1, from 23 August to 9 September 2002 (Abbott et al 2003a, for the LIGO Scientific Collaboration). Although these instruments were still to reach their design sensitivities, their performances during this period were sufficiently good to justify a serious test of our search algorithms on real interferometer data. Code to search for continuous gravitational waves from rapidly rotating neutron stars has been under development within the LIGO Scientific Collaboration (LSC) since the mid- to late-1990s, and for S1 the LSC pulsar upper limits group (PULG) used two independent search methods to set upper limits on signals from the millisecond pulsar PSR J1939+2134. This object has the shortest spin period of any known pulsar and falls in a relatively quiet part of the detector bands. Its short period also enables 


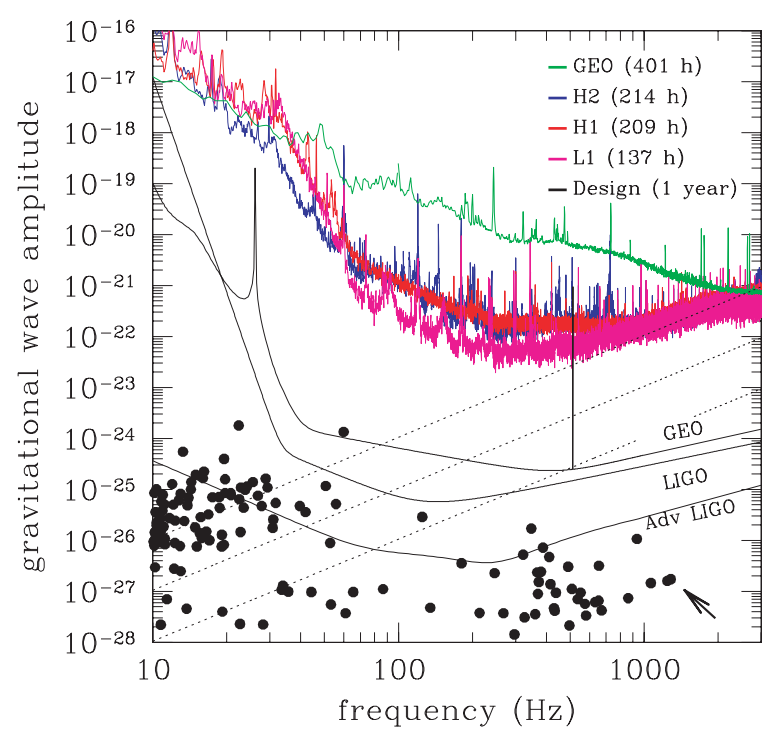

Figure 1. Mean upper limits, as a function of signal frequency, for the characteristic amplitude of gravitational waves from rotating neutron stars as constrained by the $\mathrm{S} 1$ data (upper curves). The lower three curves represent 1 year design sensitivities for GEO, LIGO and one possible design for Advanced LIGO. The dotted lines show strain amplitudes corresponding to neutron stars at $8.5 \mathrm{kpc}$ with equatorial ellipticities of $10^{-3}, 10^{-4}$ and $10^{-5}$. The dots show characteristic amplitudes from known pulsars assuming all the observed loss in rotational kinetic energy is dissipated in gravitational waves. The arrow points to PSR J1939+2134.

(This figure is in colour only in the electronic version)

us to place a relatively tight upper limit on the equatorial ellipticity of the neutron star. Here we present a brief outline of the results from this exercise. A more detailed presentation of the methods and discussion of the results can be found in Abbott et al (2003b) (for the LIGO Scientific Collaboration).

\section{Detector sensitivities}

In setting an upper limit on the gravitational wave amplitude from a pulsar we are saying something about the pulsar's physical parameters as constrained by instrumental performance rather than simply making a statement about the strain sensitivity of our instrument. The strength of any continuous gravitational wave signal from PSR J1939+2134 seen by a detecting interferometer will depend not only on the pulsar's distance, quadrupole moment and spin period, but also on the relative orientation between its axis of spin and the arms of the interferometer. The diurnal motion of the pulsar through the antenna pattern will also modulate the strength of the signal, so there is no simple relationship between the strain sensitivity curve of an interferometer and the upper limit that interferometer can place on the parameters of a particular pulsar. We can however define a mean performance, averaged over these unknowns, for a particular dataset and detection method. Figure 1 shows how our interferometers constrain monochromatic strain amplitudes at Earth as a function of signal frequency, averaged over the antenna pattern and relative orientation of the pulsar spin axis to the interferometer. The curves are derived from the 'frequency domain method' described below, and represent detection with a $1 \%$ false alarm rate and a $10 \%$ false dismissal rate using the actual observation times and data from S1. The lower solid curves show the design sensitivities of the interferometers for 
a 1 year observation period. The dotted lines indicate the strain amplitudes corresponding to hypothetical neutron stars at $8.5 \mathrm{kpc}$ with equatorial ellipticities of $10^{-3}, 10^{-4}$ and $10^{-5}$. Finally the dots represent the upper limit on strain amplitude for a selection of pulsars. For this we have assumed a simple spindown model in which all the apparent loss in rotational kinetic energy of the neutron star goes into the emission of gravitational waves. It is clear from this figure that no detection was expected from the $\mathrm{S} 1$ data. Our primary aims in this exercise were to test the data reduction and analysis methods in the presence of realistic interferometer noise and set an upper limit on the strengths of such waves.

\section{Search methods}

Although the location, period and period derivative of PSR J1939+2134 are known to high precision the inclination of the neutron star's spin axis to the line of sight $(\iota)$ and the phase $(\phi)$, polarization $(\psi)$ and, of course, amplitude $\left(h_{0}\right)$ of the gravitational wave are unknown. All these factors affect the form of the signal and were searched over in S1 using two independent methods.

The 'frequency domain method' uses Fourier transform techniques to set a frequentist upper limit on the strain amplitude from the pulsar. The input time series is broken down into $60 \mathrm{~s}$ duration short Fourier transforms (SFTs) which are high pass filtered at $100 \mathrm{~Hz}$, Tukey windowed in the time domain and amplitude calibrated once per minute. These SFTs are then optimally combined to take account of the Doppler evolution and spindown of the signal using a Dirichlet kernel method (Jaranowski et al 1998) and used to compute a detection statistic, denoted $\mathcal{F}$, which is the likelihood ratio of the data, maximized over $\iota, \phi$ and $\psi$. Monte Carlo injections into the data stream are then used to calculate the probability distribution function (pdf) of $\mathcal{F}$. The upper limit is defined such that had a signal at the upper limit level or greater been present in an ensemble of similar experiments to the one performed, $95 \%$ of the experiments would yield values of $\mathcal{F}$ that exceed the one seen in the real experiment. The computing requirement for this Monte Carlo work is extensive, and the searches ran off-line on the Medusa cluster at UWM (296 single $1 \mathrm{GHz}$ CPU nodes and $58 \mathrm{~TB}$ of disk space) and the Merlin cluster at AEI Potsdam (180 dual $1.4 \mathrm{GHz}$ CPU nodes and $36 \mathrm{~TB}$ of disk space).

The 'time domain method' is an algorithm specifically developed to search for neutron stars with a complex, but known, rotational phase evolution. It uses heterodyne methods to reduce and filter the data to a rate of one complex value every $60 \mathrm{~s}$, estimating the noise variance over the same period to take account of non-stationarity in the data. The algorithm proceeds to carry out the search as a standard Bayesian modelling problem, determining a posterior pdf for the unknown source parameters $h_{0}, \iota, \phi$ and $\psi$. The prior for $h_{0}$ is chosen to be uniform over $[0, \infty]$ and 0 for $h_{0}<0$, and the three other priors are chosen to be least informative about the orientation of the neutron star. The time domain Bayesian upper limit is set via the posterior pdf for $h_{0}$, marginalized over the nuisance parameters $\iota, \phi$ and $\psi$. We define the $95 \%$ upper credible limit for $h_{0}$ as the value at which the cumulative probability of $h_{0}$ (from zero) is 0.95 .

Note that there is nothing special about the choice of domain (frequency or time) for the frequentist and Bayesian analyses - the above choice was influenced by the desire to have two very different approaches to solving the same problem.

\section{Results}

As expected the S1 data provided no evidence for continuous wave emission from PSR J1939+2134 at twice its rotation frequency (as determined by radio observations). However, 
Table 1. Summary of the $95 \%$ upper limits delivered by the two analysis methods when applied to the four interferometers taking part in S1. GEO is the UK/German GEO 600 interferometer in Hanover, LLO is the LIGO instrument in Livingston, LA. LHO-2k and LHO-4k are the two LIGO interferometers co-sited at Hanford, WA.

\begin{tabular}{lll}
\hline Instrument & Frequentist UL & Bayesian UL \\
\hline GEO & $(1.9 \pm 0.1) \times 10^{-21}$ & $(2.2 \pm 0.1) \times 10^{-21}$ \\
LLO & $(2.7 \pm 0.3) \times 10^{-22}$ & $(1.4 \pm 0.1) \times 10^{-22}$ \\
LHO-2k & $(5.4 \pm 0.6) \times 10^{-22}$ & $(3.3 \pm 0.3) \times 10^{-22}$ \\
LHO-4k & $(4.0 \pm 0.5) \times 10^{-22}$ & $(2.4 \pm 0.2) \times 10^{-22}$ \\
\hline
\end{tabular}

we were able to improve the best upper limit for such signals by about a factor of 100 , based on earlier work by Hough et al (1983) and Hereld (1983).

The results from the four interferometers that took part in S1 are summarized in table 1, the quoted uncertainties being largely due to calibration. The two analysis methods are very different, and the meaning of 'upper limit' is not the same for both, however, we hope that the concept of an upper limit is sufficiently robust that the two interpretations would deliver numerically similar results, as indeed they do. In principle, the Bayesian analysis allows the data from all four interferometers to be combined to give an improved joint upper limit, although the relative phase calibration of the instruments was not sufficiently reliable to do this for S1. It is, however, interesting to note that an upper limit for $h_{0}$ can, within a suitable model, be interpreted as an upper limit on the equatorial ellipticity of the neutron star. If we take the distance to the pulsar to be $3.6 \mathrm{kpc}$, its radius to be $10 \mathrm{~km}$ and its mass to be $1.4 M_{\odot}$, a value of $h_{0}<1.4 \times 10^{-22}$ corresponds to an ellipticity of less than $2.7 \times 10^{-4}$. Such a large upper limit to the ellipticity could be rejected a priori, as it implies a much greater spindown rate (via gravitational luminosity) than is seen in this pulsar. However as detector sensitivities improve we can expect the above methodology to deliver constraints on neutron star parameters not otherwise achievable.

\section{Acknowledgments}

The authors gratefully acknowledge the support of the United States National Science Foundation for the construction and operation of the LIGO Laboratory and the Particle Physics and Astronomy Research Council of the United Kingdom, the Max-Planck-Society and the State of Niedersachsen/Germany for support of the construction and operation of the GEO 600 detector. The authors also gratefully acknowledge the support of the research by these agencies and by the Australian Research Council, the Natural Sciences and Engineering Research Council of Canada, the Council of Scientific and Industrial Research of India, the Department of Science and Technology of India, the Spanish Ministerio de Ciencia y Tecnologia, the John Simon Guggenheim Foundation, the David and Lucile Packard Foundation, the Research Corporation and the Alfred P Sloan Foundation.

\section{References}

Abbott B et al (The LIGO Scientific Collaboration) 2003a Preprint gr-qc/0308043

Abbott B et al (The LIGO Scientific Collaboration) 2004 Phys. Rev. D 64 at press (Preprint gr-qc/0308050)

Hough J et al 1983 Nature $\mathbf{3 0 3} 216$

Hereld M 1983 PhD Dissertation California Institute of Technology

Jaranowski P, Królak A and Schutz B F 1998 Phys. Rev. D 58063001 